\title{
Relationship of the P Angle with Stereotactic Arc in Intraoperative Outcome of Patients with Parkinson's Disease Undergoing Deep Brain Stimulation
}

\author{
Mansour Parvaresh ${ }^{1}$, Maziar Azar ${ }^{2}$, Babak Alijani ${ }^{3}$ * \\ ${ }^{1}$ MD, Assistant Professor of Neurosurgery, Neurosurgery Department, Iran University of Medical Sciences, Tehran, Iran \\ ${ }^{2}$ MD, Associate Professor of Neurosurgery, Neurosurgery Department, Iran University of Medical Sciences, Tehran, Iran \\ ${ }^{3}$ MD, Assistant Professor of Neurosurgery, Neurosurgery Department, Poursina Hospital, Guilan University of Medical Sciences, \\ Rasht, Guilan, Iran \\ * Corresponding Author Address: Poursina Hospital, Guilan University of Medical Sciences, Rasht, Guilan, Iran. Tel: +981333368773, \\ Fax:+981333339842. E-mail: babakalij@gmail.com
}

Article Type: Research Article

Received: December 8, 2015, Last revised: December 12, 2015, Accepted: December 19, 2015

\section{Abstract}

Background \& Aim: DBS (deep brain stimulation) is a new and successful technique in treatment of symptoms of Parkinsonism especially after drug resistance. Research in this field is mostly designed for evolution of this technique. The present study aimed at evaluating the relationship between the angle formed in midsagittal and STN (sub-thalamic nucleus) axis line and recording length in the final electrode placement.

Methods \& Materials/Patients: 46 patient candidates for DBS operation were studied in terms of demographic variables, STN nucleus length, the angle between midsagittal line and STN axis (p angle), the number of tested electrodes, force and length of final electrode registration and final coordinates of the placed electrode. The primary information was obtained from patients records and other technical information based on MRI imaging using Stereonata software and during surgery. The information were analyzed using SPSS (version 16) and descriptive analysis and linear relationship.

Results: The mean force of the recording from trial microelectrodes implanted in the right side ranged from $1.49 \pm$ 1.45 to $2.65 \pm 1.42$ and in the left side from $1.53 \pm 1.35$ to $2.65 \pm 2.30$. In comparative analysis, no significant statistical relationship was found between $\mathrm{P}$ angle of the right side and degree registered in the final electrode of the right side (Pearson correlation: 0.314 , $\mathrm{P}$ value $=0.049$ )

Conclusion: Not only accurate electrodes positioning in the STN can lead to improved outcome within bilateral STN DBS, but also optimizing defined P angle can have beneficial effects on intraoperative outcome after STN DBS.

Keywords: Sub-thalamic Nucleus Axis; Mid-sagittal Line; Stereotactic; T2-weighted Coronal; Intraoperative Outcome; Parkinson, Deep Brain Stimulation

Please cite this paper as: Parvaresh M, Azar M, Alijani B. Relationship of the P Angle with Stereotactic Arc in Intraoperative Outcome of Patients with Parkinson's Disease undergoing Deep Brain Stimulation. Iran. J. Neurosurg. 2015;1(3):6-10.

\section{Introduction}

Recent improvements in various surgical procedures for treating parkinson's disease (PD) has led to a significant reduction in the operative risk. Deep brain stimulation of the subthalamic nucleus (STN-DBS) is currently accepted as a highly effective surgical treatment for PD which complicated by dyskinesia, disability and idiopathic motor fluctuation (1-4). The best management of STNDBS should be relied on maximizing benefit and minimizing risk (5). In some recent studies, the improvement of short-term and long-term outcomes including increase in daily living activities and motor functions as well as lower medication load and its-related side effects has been described (4). Furthermore, it has been shown that the use of this surgical technique results in weakened clinical manifestations such as tremor, bradykinesia, rigidity and dystonia (6-9). Different randomized studies suggested that better surgical outcome can be obtained in PD patients with medication-refractory and difficult to control onoff fluctuations (9-11).

Although there is a general agreement for the efficacy of STNDBS in removing PD symptoms, selecting the best protocols for
DBS lead placement is still controversial (1). One of the most important components of DBS implantation surgery technique is sterotactic anatomic targeting and target verification. A variety of approaches are available to localize optimal target. Anatomical targeting was initially applied to achieve the most precise localization. Primarily, the stereotactic coordinates of the anterior commissure (AC) and posterior commissure (PC) lines were used in indirect targeting $(12,13)$. However, with the advances in neuroimaging technologies, direct visualization of the various nuclei is now possible, so that it can offer excellent stereotactic precision. In this regard, magnetic resonance imaging (MRI) can offer the best anatomical resolution in multiple plans (4). On the other hand, the STN on coronal T2-weighted images is easily identified (14-16) and can be localized and inserted by DBS leads during surgery.

The position of the stimulator contact of the DBS electrode is one of the important factors for achieving optimal clinical results $(4,6,9)$. However, it is difficult to understand the anatomical relationship between the electrode and the STN based on an atlas. For the first time, the angle between STN axis and mid- 
sagittal line was calculated to determine the best trajectory. The angle of the electrode trajectory is about 60 degrees from the horizontal plane of the AC-PC line and about 20 degrees from the sagittal plane (17).

In the present study, we first aimed to estimate the angle made between STN axis and mid-sagittal line (as angle $\Theta)$ and estimate the angle of difference between $\Theta$ and the stereotactic arc (as P angle) during targeting process. Then, we purposed to determine the relationship between $\mathrm{P}$ angle and study endpoint defined as the recorded length and force from final fixed microelectrodes on both left and right sides. To our knowledge, this is the first innovative attempt that assays these relations worldwide (Figure 1).

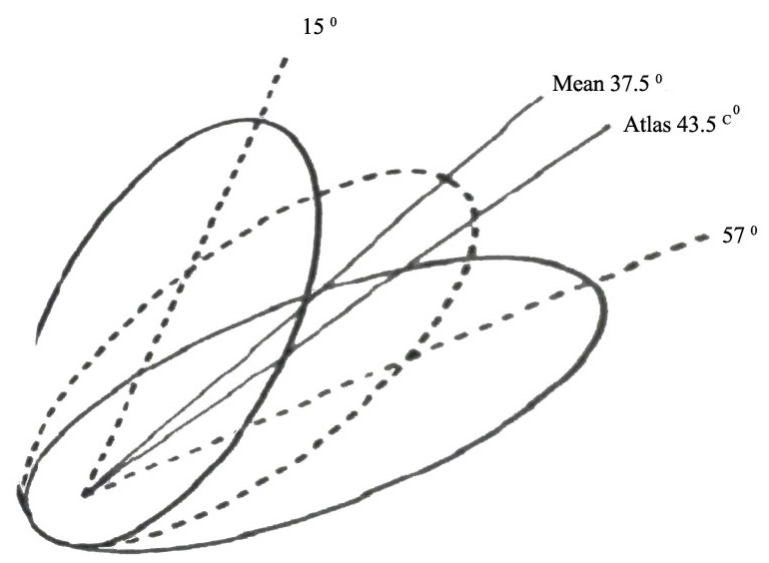

Figure 1. Schematic View of STN angle from Mid Control Line

\section{Methods and Materials}

In a prospective study within the three years between 2006 and 2009, 92 DBS electrodes were implanted in 46 patients with the final diagnosis of PD referred to Parkinson clinic of Rasoul-e-Akram University Hospital in Tehran, Iran. All patients had bilateral implantation in the same operation. PD in all participants was diagnosed based on the modified specific clinical criteria of PD reported by The United Kingdom Brain Bank (18). All patients received appropriate medical control by neurologists in our university hospital prior to the surgical treatment. Those with contraindications for surgery including impaired general status, history of psychiatric disorders, heart failure, taking anticoagulants, pacemakers in sentinel mode or dementia were not included. The study protocol was approved by the institutional board, and informed consent was obtained from all patients because their surgical and clinical data may be used in our research. Baseline characteristics and clinical data were collected from hospital recorded file including age, disease duration, medications and dominant site of symptom. Technically, the information was obtained based on MRI plane. A Leksell-G stereotactic frame was applied under local or general anesthesia. We fixed the frame parallel to the orbitomeatal axis and then patients were taken to the MRI unit where special sequences were obtained by a 1.5 Tesla machine (Philips). DBS and MRI parameters such as target coordinates $(\mathrm{X}, \mathrm{Y}, \mathrm{Z})$ in all three axes on both sides were reported and calculated by three methods of manual, use of SchaltenbrandWahren stereotactic (S-W) atlas and stereotactic software (Steronata). The series of images were taken pre-operatively by MR T2 weighted coronal view to better define the STN. The target coordinates were reported to the T2-weighted image in order to find the best trajectories for insertion of DBS electrodes. The CRW arc and ring angles relative to the ACPC plane were determined. After the STN defining, the angle between STN axis and mid-sagittal line was determined (angle $\Theta)$ for both sides. Sterotactic arc was also determined using Steronata software based on the best trajectory we determined. Then, the angle of difference between $\Theta$ and the stereotactic arc (as P angle) was calculated as: (Figure 2)

$$
\mathrm{P}=\mid \text { 90-Arc } \mid-\Theta
$$

In the operating room, we tried to insert 5 trial microelectrode (ant, post, central, med and lat), then the force and the length of each trial microelectrode were recorded and pointed from zero (flat line) to four (best STN recording amplitude), subsequently the most appropriate force and length were obtained bilaterally. Then we made stimulation on each electrode and compared the clinical responses (reduction of bradykinesia and tremor with the least side effects). At last, we determined the best trajectory with higher recording data and appropriate clinical responses without side effects (Figure 3).

Our study endpoint was to clarifying the relation between $\mathrm{P}$ angle values and two parameters of the recording force and the length of recording from final fixed microelectrodes intra operatively. For statistical analysis, results were presented as mean \pm standard deviation (SD) for quantitative variables and were summarized by absolute frequencies and percentages for categorical variables. Correlation between the parameters was examined using the Pearson's correlation coefficient test. Statistical significance was determined as the $P$ value $<0.05$. All statistical analysis were performed using SPSS software (version 20.0, SPSS lnc., Chicago, Illinois).

\section{Results}

The mean age of patients was about $49.93 \pm 8.94$ years (ranged from 31 to 72 years) of whom, 37 (80.0\%) were male. The mean duration of disease was also $11.17 \pm 5.05$ years (ranged from 5 to 25 years). For each STN, we inserted five microelectrode (anterior, posterior, medial, lateral and central according to the anatomic position of nucleus) and after recording and stimulation the better amplitude of recording which had the best clinical response (reduction of bradykinesia and tremor) was determined as the final trajectory for the permanent lead implantation track. Tracks chosen for permanent microelectrode implantation in the right side included $9(20.9 \%)$ posterior tracks, $17(39.5 \%)$ central tracks, and $10(23.3 \%)$ lateral tracks and for the left side included $7(16.3 \%)$ posterior tracks, 18 (41.9\%) central tracks, $13(30.2 \%)$ anterior tracks, 4 (9.3\%) medial tracks and $1(2.3 \%)$ lateral track. The mean number of implanted electrodes with effective recording was 3.5. For all procedures in which the STN was visualized (43 patients), the mean distance ranges of the AC/PC-based from the center of STN were as follows: $5.73 \pm 1.09 \mathrm{~mm}$ (range of 1-6 mm) in the right side and $5.59 \pm 1.44 \mathrm{~mm}$ (range of $1.5-8.0 \mathrm{~mm}$ ) in the left side. As shown in Table 1, the mean force of the recording from trial microelectrodes implanted in the right side ranged from $1.49 \pm 1.45$ to $2.65 \pm 1.42$ and in the left side from $1.53 \pm$ 1.35 to $2.65 \pm 2.30$. Also, the mean length of the recording trial microelectrodes implanted in the right side ranged from $2.54 \pm$ $2.53 \mathrm{~mm}$ to $4.06 \pm 2.23 \mathrm{~mm}$ and in the left side from $2.12 \pm 2.18$ $\mathrm{mm}$ to $4.17 \pm 2.04 \mathrm{~mm}$. The descriptive characteristics of the location of both right and left STNs in X-, Y- and Z-coordinates are listed in Table 2. The mean values of X-coordinates of right and left STN were $89.06 \pm 1.53 \mathrm{~mm}$ and $111.2 \pm 1.51$ $\mathrm{mm}$, respectively. The mean values of Y-coordinates of right 

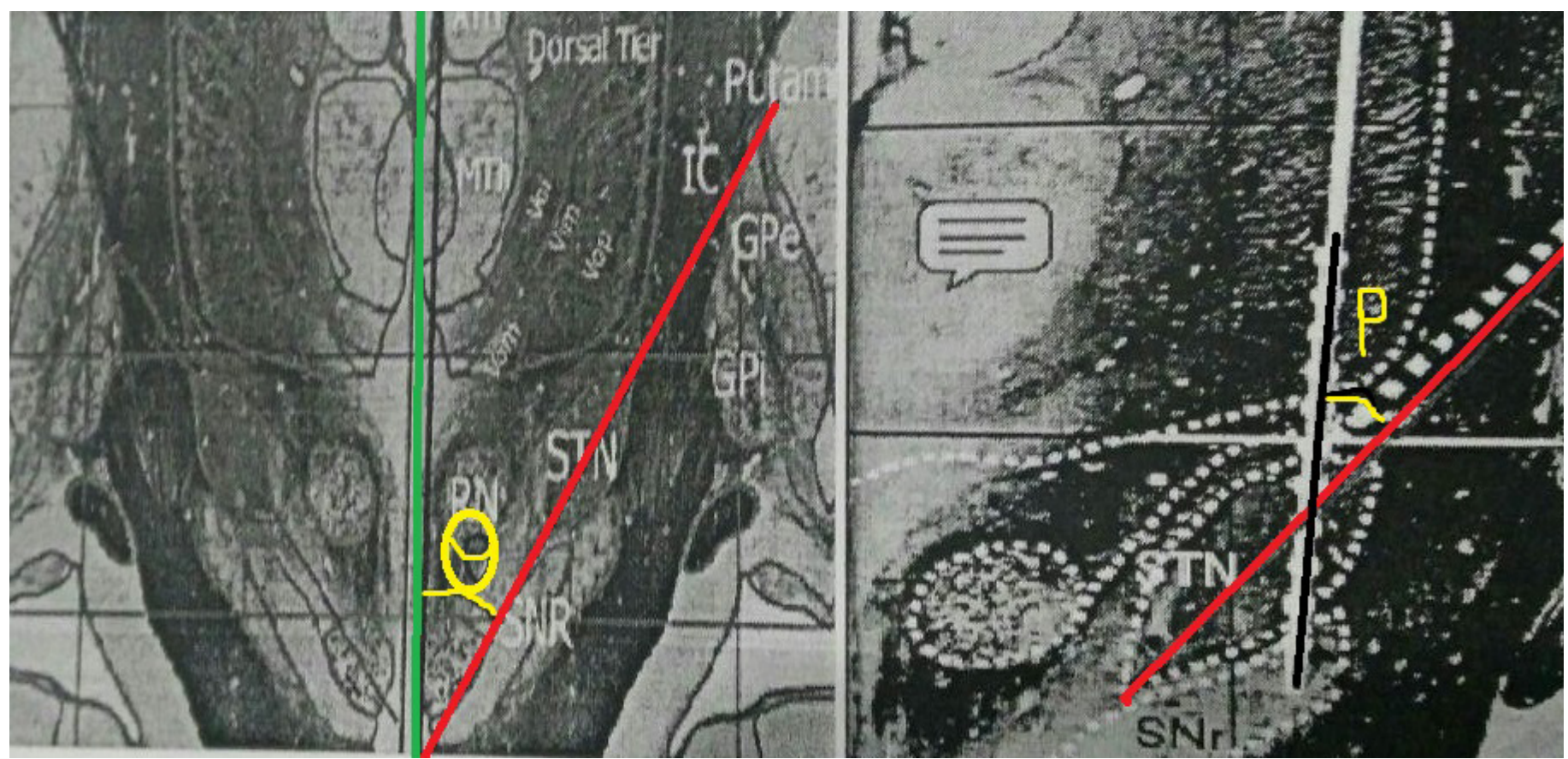

Figure 2. Anatomic View of STN with Direction of DBS leads

and left STN were $95.87 \pm 3.18 \mathrm{~mm}$ and $95.88 \pm 3.16 \mathrm{~mm}$, respectively. These values for $\mathrm{Z}$-coordinates of right and left STN were $100.16 \pm 5.44 \mathrm{~mm}$ and $100.17 \pm 5.48 \mathrm{~mm}$, respectively. In the right side, the arc and ring angles relative to the AC-PC plane ranged from $68.4 \pm 2.66$ degrees to $53.9 \pm 5.25$ degrees, respectively. Also, in the left side, the arc and ring angles relative to the AC-PC plane were in the ranges from $111.41 \pm 2.79$ to 52.81 \pm 5.7 degrees, respectively (Table 3 ). According to the definition in the method section for estimating $\Theta$ angle, the mean of this angle in the right side was $42.22 \pm 3.49$ degrees (ranged from 34.4 to 51.3 degrees) and in the left side was $42.88 \pm 3.45$ degrees (ranged from 38.0 to 56.5 degrees) but there was no significant relationship between age, sex and teta angle $(p=0.11$ for RT and $p=0.08$ for LT side. There was also no relationship between length of disease and teta angle ( $\mathrm{RT}, \mathrm{P}=0.7 \& \mathrm{LT}, \mathrm{P}=0.9)$.
Overall, the value of $\mathrm{P}$ angle was calculated based on the values of arc and $\Theta$ angles. The mean $P$ angle in the right side was 20.8 \pm 4.53 degrees (ranged from 12.4 to 30.0 degrees) and in the left side was $21.7 \pm 4.11$ (ranged from 13.7 to 33.5 degrees).

Regarding the study endpoint to determine the correlation between $\mathrm{P}$ angle values and two parameters of the recording force and length from final microelectrodes, this significant correlation was observed only between the value of defined $\mathrm{P}$ angle and recording in the right side. On the other hand, decreasing the value of $\mathrm{P}$ angle led conflicted with force of the recording and therefore $\mathrm{P}$ angle tending to zero could lead to change force of the recording. However, the relationship between the value of this angle and the length of recording in both sides were insignificant ( $\mathrm{RT} \mathrm{P}=0.135$ \& LT $\mathrm{P}=-0.84$ ).
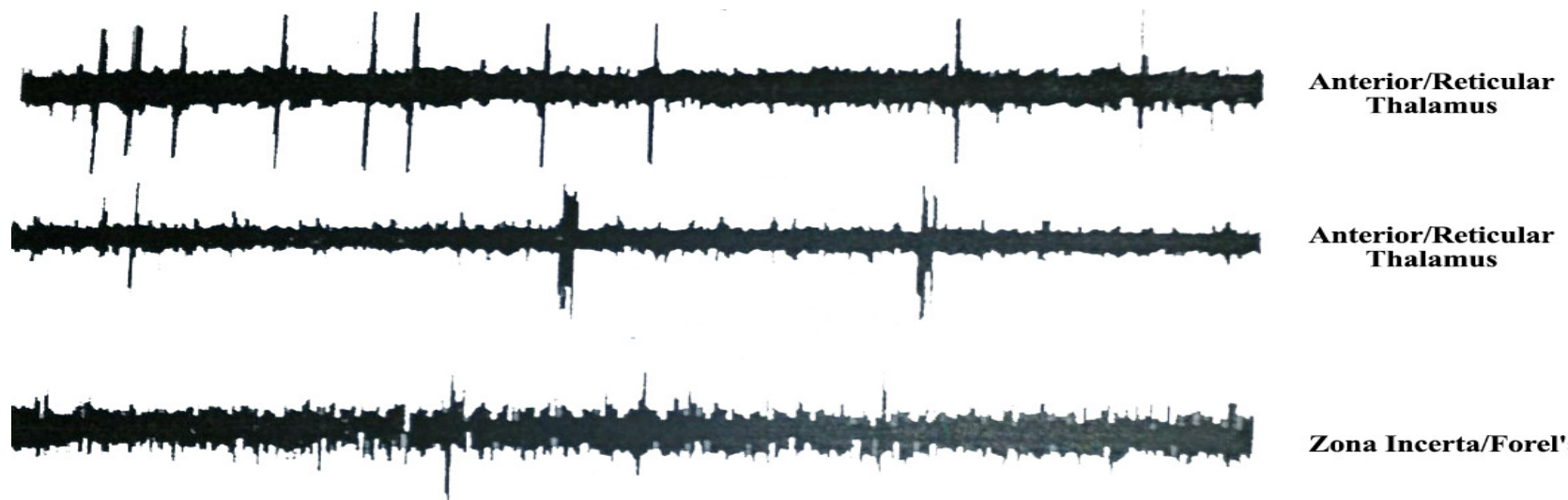

Zona Incerta/Forel's Fields

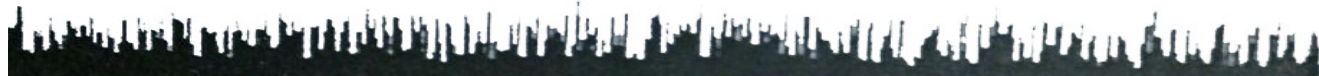

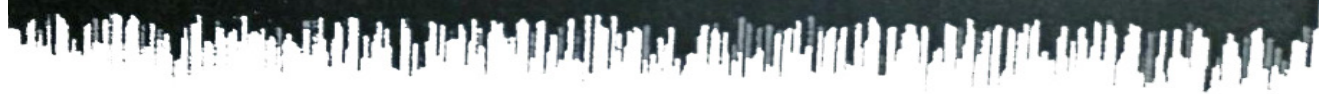

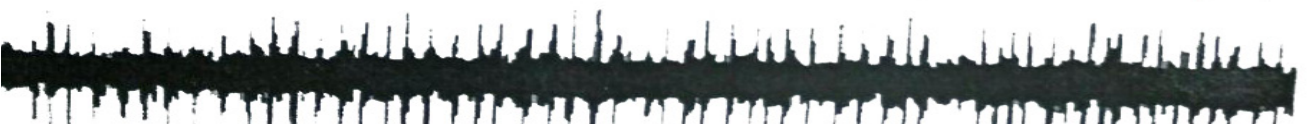

STN

SNR

Figure 3. Specific Recording Data for each Element during DBS 
Table 1. The Mean Force and Length Recorded from Microelectrodes Implanted in the Left and Right Sides

\begin{tabular}{|c|c|c|c|c|c|c|}
\hline \multirow[t]{2}{*}{ Item } & \multicolumn{3}{|c|}{$\begin{array}{l}\text { Right } \\
\text { Side }\end{array}$} & \multicolumn{3}{|c|}{$\begin{array}{l}\text { Left } \\
\text { Side }\end{array}$} \\
\hline & Mean & SD & $\begin{array}{l}\text { Range } \\
\text { from } 0\end{array}$ & Mean & SD & $\begin{array}{l}\text { Range } \\
\text { from } 0\end{array}$ \\
\hline \multicolumn{7}{|l|}{ Force } \\
\hline Anterior & 2.12 & 1.65 & 4.0 & 2.07 & 1.48 & 4.0 \\
\hline Central & 2.65 & 1.42 & 4.0 & 2.65 & 1.30 & 4.0 \\
\hline Posteror & 0.19 & 1.43 & 4.0 & 1.88 & 1.48 & 4.0 \\
\hline Medial & 1.95 & 1.51 & 4.0 & 1.72 & 1.50 & 4.0 \\
\hline Lateral & 1.49 & 1.49 & 4.0 & 1.53 & 1.35 & 4.0 \\
\hline \multicolumn{7}{|l|}{ Length } \\
\hline Anterior & 3.20 & 2.60 & 8.0 & 3.66 & 2.77 & 9.0 \\
\hline Central & 4.06 & 2.23 & 7.0 & 4.17 & 2.04 & 7.0 \\
\hline Posterior & 3.36 & 2.20 & 6.5 & 2.77 & 2.41 & 7.0 \\
\hline Medial & 3.09 & 2.37 & 7.0 & 2.12 & 2.18 & 7.0 \\
\hline Lateral & 2.54 & 2.53 & 7.5 & 2.37 & 2.23 & 6.5 \\
\hline
\end{tabular}

Table 2. The Mean Force and Length Recorded from Microelectrodes Implanted in the Left and Right Sides

\begin{tabular}{|c|c|c|c|c|c|c|}
\hline \multirow[t]{2}{*}{ Measures } & \multicolumn{3}{|c|}{$\begin{array}{l}\text { Right } \\
\text { Side }\end{array}$} & \multicolumn{3}{|c|}{$\begin{array}{l}\text { Left } \\
\text { Side }\end{array}$} \\
\hline & $\mathbf{X}$ & $\mathbf{Y}$ & $\mathbf{Z}$ & $\mathbf{X}$ & $\mathbf{Y}$ & $\mathbf{Z}$ \\
\hline Mean & 89.06 & 95.87 & 100.16 & 111.2 & 95.88 & 100.17 \\
\hline $\begin{array}{l}\text { Standard } \\
\text { Deviation }\end{array}$ & 1.53 & 3.18 & 5.44 & 1.51 & 3.16 & 5.48 \\
\hline Median & 7.0 & 16.0 & 27.0 & 7.0 & 15.0 & 28.0 \\
\hline Maximum & 86.5 & 90.0 & 84.5 & 109.0 & 90.0 & 84.5 \\
\hline Minimum & 93.5 & 106.0 & 111.5 & 116.0 & 105.0 & 112.5 \\
\hline \multicolumn{7}{|l|}{ Length } \\
\hline Anterior & 3.20 & 2.60 & 8.0 & 3.66 & 2.77 & 9.0 \\
\hline Central & 4.06 & 2.23 & 7.0 & 4.17 & 2.04 & 7.0 \\
\hline Posterior & 3.36 & 2.20 & 6.5 & 2.77 & 2.41 & 7.0 \\
\hline Medial & 3.09 & 2.37 & 7.0 & 2.12 & 2.18 & 7.0 \\
\hline Lateral & 2.54 & 2.53 & 7.5 & 2.37 & 2.23 & 6.5 \\
\hline
\end{tabular}

Table 3. The Range of the Arc and Ring Angles Relative to the AC-PC Plane

\begin{tabular}{|lcccc|}
\hline Measures & \multicolumn{2}{c}{ Right Side } & \multicolumn{2}{c|}{ Left Side } \\
& Arc & Ring & Arc & Ring \\
\hline Mean & 68.4 & 53.09 & 111.41 & 52.81 \\
\hline $\begin{array}{l}\text { Standard } \\
\text { Deviation }\end{array}$ & 2.66 & 5.25 & 2.79 & 5.70 \\
Median & 11.5 & 20.6 & 13.7 & 24.0 \\
\hline Maximum & 61.5 & 44.0 & 103.0 & 42.0 \\
\hline Minimum & 116.7 & 64.6 & 116.7 & 66.0 \\
\hline
\end{tabular}

\section{Discussion}

The topographical information of deep brain structures is essential for accurate stereotactic placement of the stimulating electrode of DBS. The target of a deep brain structure is usually based on the (S-W) atlas that consists of two-dimensional images such as a coronal section. It is difficult to understand localization and the shape of deep brain structures spatially, especially of the subthalamic nucleus on S-W atlas. Besides, one of the most important problems for STN-DBS is proper insertion into the STN which can lead to the optimal results. In this context, the three-dimensional image is useful for diagnosis of STN anatomy and preoperative planning as well as giving the vivid spatial image of brain complex structures (19). In addition, the postoperative clinical benefits of STN-DBS are directly related to the microelectrode placement (1). In fact, considering both clinical variables and electrophysiological recording data are mandatory to ensure optimal placement of the electrodes (16, 20, 21). Previously, the association between DBS electrode place and clinical outcome was studied (22). In fact, the principal cause of the poor clinical results arises from imprecision of electrode placement, leading to non-stimulation of the target as required. On the other hand, suitable implantation of electrodes in the STN can produce improvement in patients with poor results from surgery and with suspected electrode misplacement based on imaging findings (23). Although relationship between surgical outcome and appropriate placement of implanted microelectrodes relative to the intended target has been evaluated in numerous studies. Few studies have been conducted to assess the association between the changes in the angle made by STN axis and mid-sagittal line and its-related defined angles and clinical efficacy of electrodes (14). Logically the less P angle, the longer length of STN recording must be obtained according to coaptation of microelectrode to the main axis of STN. The findings in this study cause the authors to suggest that a change in the value of defined $\mathrm{P}$ angle can result in optimization of the force recorded from the electrodes, although we couldn't statistically prove the relationship between length of recording and $\mathrm{P}$ angle. The complimentary researches with more cases and more definite recording may clarify new results. Changes in the force of the recording intra operatively can be due to crossing the physiologic center of STN by the microelectrode. To our knowledge, this is the first study regarding determining STN angles and their role intraoperative outcomes defined on the recorded force and length from electrodes. However, we could obtain this result only in the right side electrodes probably because of small study sample size which made inaccurate results.

Totally, it can be concluded that not only accurate electrodes positioning in the STN can lead to improved outcome within bilateral STN DBS, but also optimizing defined P angle can have beneficial effects on intraoperative outcome after STN DBS. However, future studies can focus on prospective evaluation of the degree to which modification of this angle determined target leading to improve clinical efficacy of DBS electrodes.

\section{Acknowledgment}

The authors would like to thank the Clinical Research Development Unit of Poursina Hospital, Guilan University of Medical Sciences, Rasht, Guilan, Iran.

\section{Funding}

None. 


\section{Conflicts of Interest}

The authors declare that they have no conflicts of interest.

\section{References}

1. Houeto J, Damier P, Bejjani P, Staedler C, Bonnet A, Arnulf I, et al. Subthalamic stimulation in Parkinson disease: a multidisciplinary approach. Archives of neurology. 2000;57(4):461-5.

2. Dostrovsky JO, Lozano AM. Mechanisms of deep brain stimulation. Movement Disorders. 2002;17(S3):S63-S8.

3. Vitek JL. Mechanisms of deep brain stimulation: excitation or inhibition. Movement disorders. 2002;17(S3):S69-S72

4. Benabid AL, Benazzous A, Pollak P. Mechanisms of deep brain stimulation. Movement Disorders. 2002;17(S3):S73-S4.

5. Okun MS, Foote KD. Parkinson's disease DBS: what, when, who and why? The time has come to tailor DBS targets. 2010.

6. Hamel W, Fietzek U, Morsnowski A, Schrader B, Herzog J, Weinert D, et al. Deep brain stimulation of the subthalamic nucleus in Parkinson's disease: evaluation of active electrode contacts. Journal of Neurology, Neurosurgery \& Psychiatry. 2003;74(8):1036 46.

7. Nunta-Aree S, Sitthinamsuwan B, Boonyapisit K, Pisarnpong A. SW2-year outcomes of subthalamic deep brain stimulation for idiopathic Parkinson's disease. J Med Assoc Thai. 2010;93(5):529-40.

8. Ford B, Winfield L, Pullman S, Frucht S, Du Y, Greene P, et al. Subthalamic nucleus stimulation in advanced Parkinson's disease: blinded assessments at one year follow up. Journal of Neurology, Neurosurgery \& Psychiatry. 2004;75(9):1255-9.

9. Harries AM, Kausar J, Roberts SA, Mocroft AP, Hodson JA, Pall HS, et al. Deep brain stimulation of the subthalamic nucleus for advanced Parkinson disease using general anesthesia: long-term results: Clinical article. Journal of neurosurgery. 2012;116(1):107-13.

10. Okun MS, Fernandez HH, Wu SS, Kirsch Darrow L, Bowers D, Bova F, et al. Cognition and mood in Parkinson's disease in subthalamic nucleus versus globus pallidus interna deep brain stimulation: the COMPARE trial. Annals of neurology. 2009;65(5):586-95

11. Follett KA, Weaver FM, Stern M, Hur K, Harris CL, Luo P, et al. Pallidal versus subthalamic deep-brain stimulation for Parkinson's disease. New England Journal of Medicine. 2010;362(22):2077-91

12. Cuny E, Guehl D, Burbaud P, Gross C, Dousset V, Rougier A. Lack of agreement between direct magnetic resonance imaging and statistical determination of a subthalamic target: the role of electrophysiological guidance. Journal of neurosurgery. 2002;97(3):591-7.

13. Hamid N, Mitchell R, Mocroft P, Westby G, Milner J, Pall H. Targeting the subthalamic nucleus for deep brain stimulation: technical approach and fusion of pre-and postoperative MR images to define accuracy of lead placement. Journal of pre-and postoperative MR images to define accuracy of
Neurology, Neurosurgery \& Psychiatry. 2005;76(3):409-14

14. Yoon MS, Munz M. Placement of deep brain stimulators into the subthalamic nucleus. Stereotactic and functional neurosurgery. 1999;72(2-4):145-9.

15. Richter EO, Hoque T, Halliday W, Lozano AM, Saint-Cyr JA. Determining the position and size of the subthalamic nucleus based on magnetic resonance imaging results in patients with advanced Parkinson disease. Journal of neurosurgery. 2004;100(3):541-6

16. Bejjani B-P, Dormont D, Pidoux B, Yelnik J, Damier P, Arnulf I, et al. Bilateral subthalamic stimulation for Parkinson's disease by using three-dimensional stereotactic magnetic resonance imaging and electrophysiological guidance. Journa of neurosurgery. 2000;92(4):615-25

17. Perozzo P, Rizzone M, Bergamasco B, Castelli L, Lanotte M, Tavella A, et al. Deep brain stimulation of the subthalamic nucleus in Parkinson's disease: comparison of preand postoperative neuropsychological evaluation. Journal of the neurological sciences. 2001;192(1):9-15.

18. Gelb DJ, Oliver E, Gilman S. Diagnostic criteria for Parkinson disease. Archives of neurology. 1999;56(1):33-9.

19. Nakano N, Taneda M. [Three-dimensional atlas of subthalamic nucleus and its adjacent structures]. No shinkei geka Neurological surgery. 2005;33(7):683-92

20. Benabid A, Benazzouz A, Hoffmann D, Limousin P, Krack P, Pollak P. Long $\square$ term electrical inhibition of deep brain targets in movement disorders. Movement Disorders 1998;13(S3):119-25.

21. Rodriguez-Oroz MC, Rodriguez M, Guridi J, Mewes K, Chockkman V, Vitek J, et al. The subthalamic nucleus in Parkinson's disease: somatotopic organization and physiological characteristics. Brain. 2001;124(9):1777-90.

22. McClelland III S, Ford B, Senatus PB, Winfield LM, Du YE, Pullman SL, et al Subthalamic stimulation for Parkinson disease: determination of electrode location necessary for clinical efficacy. Neurosurgical focus. 2005;19(5):1-9.

23. Paek SH, Han JH, Lee J-Y, Kim C, Jeon BS, Kim DG. Electrode position determined by fused images of preoperative and postoperative magnetic resonance imaging and surgical outcome after subthalamic nucleus deep brain stimulation. Neurosurgery. 2008;63(5):925-37.

\section{Comments}

This was an interesting and precise research and the colleagues at Rasoul-e-Akram Hospital did an interesting and extraordinary work on this research. Their time and effort is appreciated by their findings. In the field of Functional Stereotactic neurosurgery, which is a new field with comparison to other sections of neurosurgery, many new researches can be done to further explore this field. Nevertheless, there are few researchers worldwide that have done/are doing research in this field, and few neurosurgeons are able to use the results of these articles in their practice. Therefore, researches such as the current one are rarely seen in the general neurosurgery journal, since it is focused on a sub-special field.

Sohrab Shahzadi, MD, Professor of Neurosurgery, Department of Neurosurgery, Shohada Tajrish Hospital, Shahid Beheshti University of Medical sciences, Tehran, Iran 